\title{
Athermal Character of Structural Phase Transitions
}

\author{
Francisco J. Pérez-Reche, Eduard Vives, Lluís Mañosa, and Antoni Planes \\ Departament d'Estructura i Constituents de la Matèria, Facultat de Física, Universitat de Barcelona, \\ Diagonal, 647, E-08028 Barcelona, Catalonia
}

(Received 21 March 2001; published 24 October 2001)

\begin{abstract}
The significance of thermal fluctuations in nucleation in structural first-order phase transitions has been examined. The prototypical case of martensitic transitions has been experimentally investigated by means of acoustic emission techniques. We propose a model based on the mean first-passage time to account for the experimental observations. Our study provides a unified framework to establish the conditions for isothermal and athermal transitions to be observed.
\end{abstract}

DOI: 10.1103/PhysRevLett.87.195701

Fluctuations are considered as essential for a phase transition to take place. However, at moderate temperatures many solids undergo first-order phase transitions which are regarded as being athermal. The kinetics of these transitions is not dominated by thermal fluctuations and, consequently, they can take place only under the change of an external parameter (stress, magnetic field, temperature, etc.) which modifies the free energy difference between high and low symmetry phases. This behavior is in contrast to thermally activated transitions for which the relaxation from a metastable state can occur at constant external conditions due to thermal fluctuations. Examples of athermal transitions are found within the families of magnetic and structural phase transitions which involve long-range interactions (dipolar, elastic, etc.). The martensitic transition (MT) undergone by many solids is an interesting case that will be the prototype system experimentally investigated in the present Letter. It is a diffusionless first-order transition [1] mainly caused by a shear mechanism [2]. Often MT is preceded by premonitory effects such as softening of long and short wavelength acoustic vibrational modes. The anharmonic coupling of these modes is at the origin of the instability of the high temperature phase [3].

In general, when a system is externally driven through a first-order phase transition, the response to the external control parameter is determined by the characteristics of the energy barriers separating the two phases. When thermal fluctuations are not relevant (athermal transition), the system remains in a given configuration as long as the state corresponds to a free energy local minimum. When driven, the system jumps towards a different configuration, once the local stability limit is reached. The path followed by the system is influenced by the existence of disorder (dislocations, grain boundaries, vacancies, local composition, atomic configuration, etc.), which controls the actual distribution of energy barriers. As the system evolves, it passes through a sequence of metastable states [4]. The kinetics is characterized by jumps (avalanches) from one metastable state to another which occur with a certain relaxation time. In a number of cases this time is power law distributed [5]. The jerky response for MT is related to sudden changes in the local strain field which are at the origin of the emission
PACS numbers: 64.60.My, 64.60.Qb, 81.30.Kf

of acoustic waves in the range from $\mathrm{kHz}$ to $\mathrm{MHz}$ [6]. This effect is the so-called acoustic emission (A.E.). Similar crackling behavior is observed in ferromagnetic systems (Barkhausen noise) [7], capillary condensation systems [8], and others [9]. In the athermal case the path followed by the system can be reproduced from cycle to cycle provided that disorder does not evolve [10].

Traditionally MT have been regarded as athermal [11]; however, recent experimental observations of the occurrence of the transition at constant temperature question this athermal character in shape-memory alloys [12]. The present Letter is aimed at providing a simple explanation for the observation of apparent athermal behavior and describing the possibility of a crossover between such a behavior and cases where thermal activation is evident. The problem is intimately related to the nucleation mechanism. Classical nucleation theories have proven to be inadequate for MT and heterogeneous nucleation models have been proposed, most of them neglecting the effect of themal fluctuations [13]. This problem is central for the understanding of MT and remains an issue of active debate $[11,13]$. We have investigated two prototype shape-memory materials undergoing a MT by detecting the A.E. generated during the transition. This technique is highly sensitive to tiny microstructural changes taking place in the sample. It is the best suited method to detect the nucleation and growth of any domain of the low temperature phase.

We have selected two single crystals with compositions $\mathrm{Cu}_{68.4} \mathrm{Al}_{27.8} \mathrm{Ni}_{3.8}$ and $\mathrm{Cu}_{68.0} \mathrm{Zn}_{16.0} \mathrm{Al}_{16.0}$. They underwent, respectively, a MT from the $\beta$ (ordered-bcc) towards $\gamma^{\prime}$ (hexagonal) and $\beta^{\prime}$ (orthorhombic) martensites. The samples were annealed for $30 \mathrm{~min}$ at $1173 \mathrm{~K}$ and then rapidly cooled down to room temperature and further annealed for several days. A large number of cycles (more than 50) were done in order to have a reproducible transformation. A.E. signals were detected by a resonant piezoelectric transducer acoustically coupled to the top flat surface of the sample. The bottom flat surface was mounted onto a $\mathrm{Cu}$ block which was cooled or heated by a Peltier element. In order to detect possible isothermal effects it is of major importance to avoid uncontrolled temperature oscillations and/or drifts. A computer-controlled 
feedback procedure ensured relative oscillations smaller than $0.01 \%$. The absolute value of the sample temperature [14] is known within $\pm 0.5 \mathrm{~K}$. A.E. signals were counted using a frequency counter with a $1 \mathrm{~s}$ gate [15]. Background noise is inherent to the detection of A.E.; this noise was quantified by performing experiments at a temperature of more than $100 \mathrm{~K}$ above $M_{s}$. The background count rate is in the range of $10 \mathrm{~Hz}$. This renders a signal-to-noise ratio better than $10^{3}$ during the MT.

Two complementary experimental procedures were followed for each studied sample. In the first case, the sample was cooled down from room temperature in a stepwise manner. Each step consisted of an isothermal plateau lasting a time $t$, followed by a cooling at $\dot{T}=1 \mathrm{~K} / \mathrm{min}$ down to the next plateau. The temperature difference between consecutive plateaux was $\Delta T$ and several values for $t$ and $\Delta T$ were tested. This procedure provided fine-tuning for the detection of any possible isothermal nucleation and growth of martensite. A typical example of the results obtained is shown in Fig. 1. For $\mathrm{Cu}-\mathrm{Zn}-\mathrm{Al}$ (Fig. 1a), no trace of A.E. was detected under isothermal holding conditions, for any value of $\Delta T$ (values as low as $\Delta T=0.2 \mathrm{~K}$ were investigated). Moreover, below a certain temperature, A.E. resumes each time the temperature is decreased. The behavior found for $\mathrm{Cu}-\mathrm{Al}-\mathrm{Ni}$ (Fig. 1b) turns out to be different. For isothermal plateaux separated by more than $0.8 \mathrm{~K}$, the behavior is similar to that reported for $\mathrm{Cu}-\mathrm{Zn}-\mathrm{Al}$. However, for smaller $\Delta T$, A.E. under isothermal conditions is detected. Furthermore, it is worth noting that in this case

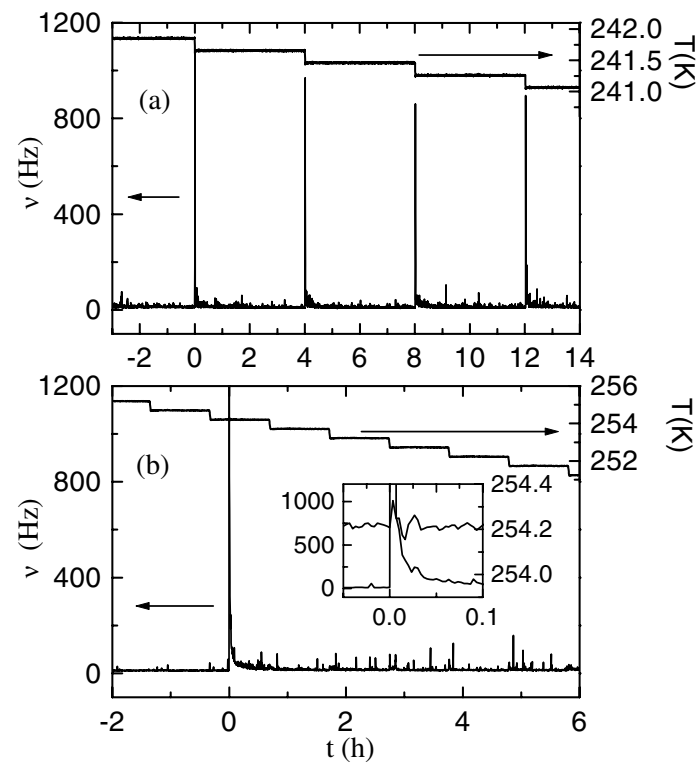

FIG. 1. Typical examples of the A.E. recorded during stepwise isothermal runs for (a) $\mathrm{Cu}-\mathrm{Zn}-\mathrm{Al}$ and (b) $\mathrm{Cu}-\mathrm{Al}-\mathrm{Ni}$. Notice the difference in the temperature scale (right axis) between the two plots. The inset of (b) shows a detailed view of the A.E. recorded isothermically. The large fluctuation in the temperature is caused by the latent heat released during the isothermal transformation of a martensitic domain.
A.E. continues for many minutes during the isothermal plateau above the average background noise. From these stepwise experiments, it can be inferred that thermal fluctuations do trigger the $\mathrm{MT}$ for $\mathrm{Cu}-\mathrm{Al}-\mathrm{Ni}$, when the system is close enough to the stability limit. In contrast, it seems that thermal fluctuations are not able to trigger the transition in $\mathrm{Cu}-\mathrm{Zn}-\mathrm{Al}$ even in the case when the system is in the two-phase coexistence region.

The second procedure consists of measuring A.E. under continuous cooling at different rates $\dot{T}$. Results for $\mathrm{Cu}-$ $\mathrm{Zn}-\mathrm{Al}$ are shown in Fig. 2a. We propose that, for athermal transitions, the absence of kinetic effects will result in a scaling of the ratio between the acoustic activity and the cooling rate $(\nu / \dot{T})$. We have evaluated the actual cooling rate at each temperature and the results for $\nu / \dot{T}$ are plotted as a function of temperature in Fig. 2b. Excellent scaling is observed over all the temperature range and a magnified view of the high temperature region is shown in the inset. The larger scatter for smaller $\dot{T}$ is due to the propagation of the error in the temperature measurement (the relative error in $\nu / \dot{T}$ is proportional to $1 / \dot{T})$. The results obtained for $\mathrm{Cu}-\mathrm{Al}-\mathrm{Ni}$ are presented in Fig. 3. No scaling is observed over all the temperature domain, thus indicating that the transition does not have an athermal character for this alloy system. Instead, the transition is not reproducible and the values for $M_{s}$ exhibit a stochastic character. The probability distribution of $M_{S}$ can be characterized

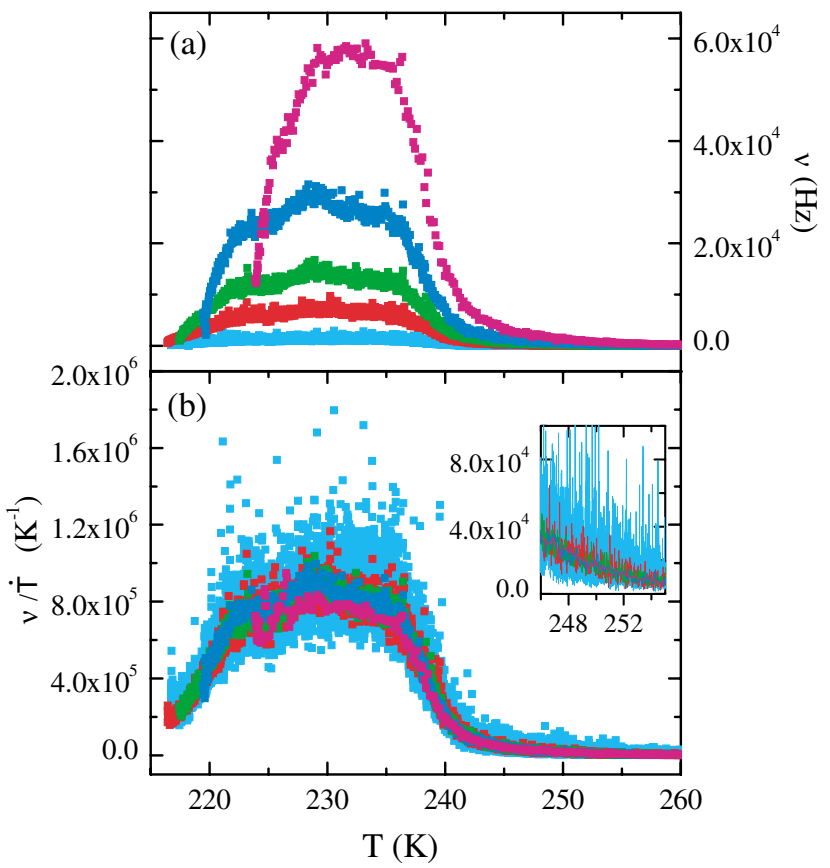

FIG. 2 (color). (a) A.E. recorded during continuous cooling runs for $\mathrm{Cu}-\mathrm{Zn}-\mathrm{Al}$ at cooling rates of $\dot{T}=5 \mathrm{~K} / \mathrm{min}$ (pink), $\dot{T}=$ $2 \mathrm{~K} / \mathrm{min}$ (dark blue), $\dot{T}=1 \mathrm{~K} / \mathrm{min}$ (green), $\dot{T}=0.5 \mathrm{~K} / \mathrm{min}$ (red), $\dot{T}=0.1 \mathrm{~K} / \mathrm{min}$ (blue). (b) Scaled A.E. as a function of temperature, showing the scaling of the different curves. The inset shows an enlarged view of the tail of the curves. 


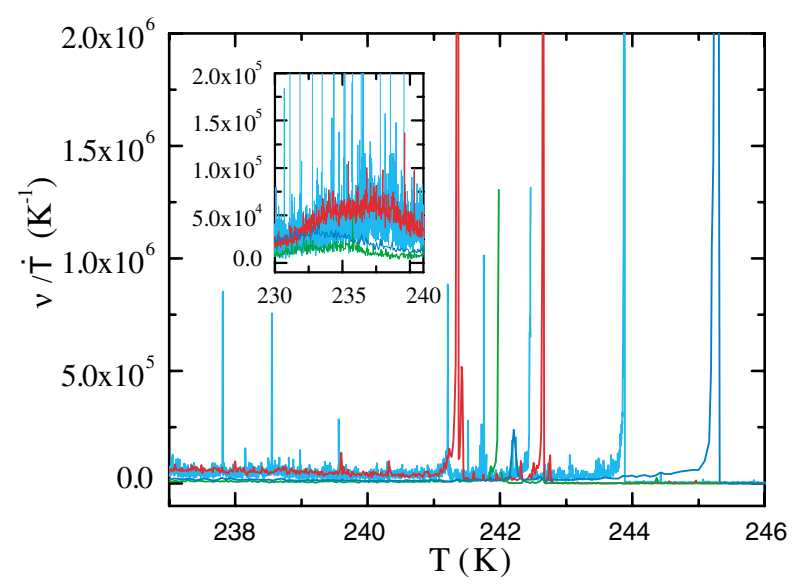

FIG. 3 (color). Scaled A.E. recorded during continuous cooling runs for $\mathrm{Cu}-\mathrm{Al}-\mathrm{Ni}$ at cooling rates of $\dot{T}=2 \mathrm{~K} / \mathrm{min}$ (dark blue), $\dot{T}=1 \mathrm{~K} / \mathrm{min}$ (green), $\dot{T}=0.5 \mathrm{~K} / \mathrm{min}$ (red), and $\dot{T}=$ $0.1 \mathrm{~K} / \mathrm{min}$ (blue). The inset shows a detail of the low temperature region.

by its average value $\left\langle M_{s}\right\rangle$ and its standard deviation $\sigma=$ $\left(\left\langle M_{s}^{2}\right\rangle-\left\langle M_{s}\right\rangle^{2}\right)^{1 / 2}$. The behavior of these two quantities as a function of $\dot{T}$ can be estimated by performing a statistical analysis of $M_{s}$ data for a large number of temperature loops. Results are shown in Fig. 4. The total number of cycles analyzed for each value of $\dot{T}$ ranges from 15 for $\dot{T}=0.5 \mathrm{~K} / \mathrm{min}$ to 150 for $\dot{T}=6 \mathrm{~K} / \mathrm{min}$.

A proper statistical analysis requires the detection and suppression of any possible systematic deviation of the data or even a fake dependence on $\dot{T}$ which may arise from ageing phenomena during the experiment. To prevent these

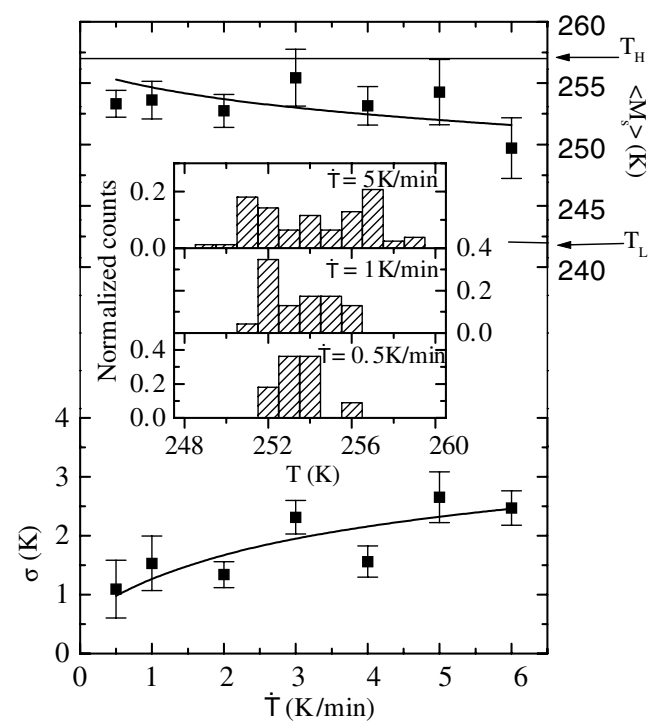

FIG. 4. Average value of the transition temperatures $\left\langle M_{s}\right\rangle$ and its standard deviations $\sigma$ (symbols), as a function of the cooling rate $\dot{T}$. Thick continuous lines correspond to the fits of the three-parameter model proposed in the text. The thin lines indicate the fitted values of $T_{H}$ and $T_{L}$. The inset shows examples of histograms of $M_{s}$ distributions. undesired effects, the experiment was performed as a series of temperature loops $(220 \mathrm{~K} \rightarrow 340 \mathrm{~K} \rightarrow 220 \mathrm{~K})$ and each series corresponded to a given value of $\dot{T}$. The series are separated by low temperature waiting periods randomly selected within the range from 1 to $1000 \mathrm{~h}$. The studied values for $\dot{T}$ within the sequence are also randomly chosen, and each value appears in the sequence several times. A small drift of $\left\langle M_{s}\right\rangle(<1.4 \mathrm{~K} /$ month $)$ with ageing time was detected and corrected from the data displayed in Fig. 4. The standard deviation $\sigma$ was found to be insensitive to ageing.

The behavior of $\left\langle M_{s}\right\rangle$ and $\sigma$ can be explained by an adequate theory based on the analysis of the mean firstpassage time [16] between the $\beta$ and the martensitic phase. Here we briefly summarize this theoretical approach. The probability $P(t)$ for the system to remain in the metastable phase after time $t$ is given by $d P(t) / d t=-\lambda(t) P(t)$, where $\lambda(t)$ is the transition probability per unit time. As for certain systems the transition seems to be athermal, while it is not in others; our main assumption in reproducing the experimental observations is that thermal fluctuations are active only within two characteristic limiting temperatures: when $T>T_{H}, \lambda=0$ (no transition is possible), and when $T<T_{L}, \lambda=\infty$ (the transition occurs with absolute certainty). This assumption leads to the following expressions for $\left\langle M_{s}\right\rangle$ and $\sigma^{2}$ :

$$
\begin{gathered}
\left\langle M_{s}\right\rangle=T_{H}-\dot{T}\langle t\rangle, \\
\sigma^{2}=\dot{T}^{2}\left\langle t^{2}\right\rangle+T_{H}^{2}-2 \dot{T} T_{H}\langle t\rangle-\left\langle M_{s}\right\rangle^{2},
\end{gathered}
$$

where the mean first-passage time $\langle t\rangle$ and $\left\langle t^{2}\right\rangle$ are given by $\dot{T}\langle t\rangle=\int_{T_{L}}^{T_{H}} e^{W(\theta)} d \theta$ and $\dot{T}^{2}\left\langle t^{2}\right\rangle=2\left(\dot{T}\langle t\rangle T_{H}-\right.$ $\left.\int_{T_{L}}^{T_{H}} \theta e^{W(\theta)} d \theta\right) . W(\theta)$ is the logarithm of the probability that the transition has not occurred before the temperature $\theta$ is reached, and is given by $W(\theta)=-\int_{\theta}^{T_{H}} \lambda(u) d u / \dot{T}$.

In order to compare the predictions of the model with the experimental results, we must propose a function $\lambda(T)$. Different functions were checked and the results obtained were qualitatively equivalent. The simplest assumption is that $\lambda$ exhibits a pole at $T_{L}$ and a zero at $T_{H}$, i.e., $\lambda=\omega\left(T_{H}-T\right) /\left(T-T_{L}\right)$, where $\omega$ is a characteristic frequency for nucleation. By inserting $\lambda$ into the expression for $W(\theta)$ above, we find

$$
W(\theta)=\frac{\omega}{\dot{T}}\left[\left(T_{H}-\theta\right)-\left(T_{H}-T_{L}\right) \ln \left(\frac{T_{H}-T_{L}}{\theta-T_{L}}\right)\right] .
$$

By a simultaneous nonlinear minimum- $\chi^{2}$ fitting to the $\left\langle M_{s}\right\rangle$ and $\sigma$ data for $\mathrm{Cu}_{68.4} \mathrm{Al}_{27.8} \mathrm{Ni}_{3.8}$, we obtain the following estimations of the three-free-parameter model: $T_{H}=$ $257 \pm 1 \mathrm{~K}, \quad T_{L}=242 \pm 1 \mathrm{~K}, \quad$ and $\quad \omega=(5.2 \pm$ $0.5) 10^{-2} \mathrm{~s}^{-1}$. The fit agrees remarkably well with the experimental data (Fig. 4) and reproduces the increase of $\sigma$ with $\dot{T}$ and also the slightly decreasing behavior of $\left\langle M_{S}\right\rangle$. The fitted values of $T_{H}$ and $T_{L}$ are estimations of the extreme values of $M_{s}$. Note that $\omega\left(T_{H}-T_{L}\right) \simeq 50 \mathrm{~K} / \mathrm{min}$ 
renders an estimation of the cooling rate above which no time effects will be observed in $\mathrm{Cu}_{68.4} \mathrm{Al}_{27.8} \mathrm{Ni}_{3.8}$. On the other hand, for the $\mathrm{Cu}_{68.0} \mathrm{Zn}_{16.0} \mathrm{Al}_{16.0}$ sample we have found athermal behavior, even at $0.1 \mathrm{~K} / \mathrm{min}$. Assuming that $\omega$ for this sample is similar to the previous one, $T_{H}-T_{L}<2 \mathrm{~K} . T_{H}$ and $T_{L}$ coincide within the errors, and no isothermal behavior can be observed with the allowed temperature control resolution.

Our results indicate that strictly, at finite temperatures, athermal transitions do not occur. However, in practice, if $T_{H}-T_{L}$ is small enough, kinetic effects are difficult to observe. In this case (close to the athermal limit), scaling of $(\nu / \dot{T})$ versus $T$ will occur for A.E. measurements. We have verified the robustness of such scaling in the case of the $\mathrm{Cu}-\mathrm{Zn}-\mathrm{Al}$. We claim that, in general, $d x / d T=(d x / d t) / \dot{T}$ versus $T$ will be independent of $\dot{T}$, where $x$ is the transformed fraction. The model proposed in this Letter refers to the nucleation of the first martensite domain in the system. The subsequent growth will be characterized by different energy barriers. For $\mathrm{Cu}-\mathrm{Zn}-\mathrm{Al}$ the fact that scaling extends well below the onset of the transition reveals that the system satisfies the conditions for it to behave athermally over all the transformation range. In contrast, scaling does not occur for $\mathrm{Cu}-\mathrm{Al}-\mathrm{Ni}$ either at the transition onset or in the subsequent evolution.

A question still remains open: why do the two selected samples, with transition temperatures within the same temperature range, have different $T_{H}-T_{L}$ ranges? We argue that the origin lies in the small symmetry differences between the corresponding low temperature phases: orthorhombic and hexagonal, which give rise to different accommodation mechanisms of the transformational shape change necessary to minimize the elastic stored energy during the transition. Microgliding and microtwinning are the operative mechanisms in $\mathrm{Cu}-\mathrm{Zn}-\mathrm{Al}$ and $\mathrm{Cu}-\mathrm{Al}-\mathrm{Ni}$, respectively [17]. Our results are consistent with the accepted idea that twinning permits a larger metastability.

To conclude, we have proposed a scaling argument to check when a first-order phase transition can be treated as athermal. The different behavior exhibited by martensites with different transformation mechanisms has been explained by a single model based on the mean firstpassage time. In the light of present results, it is clear that for systems with a lack of scaling, the definition of a single transition temperature $M_{s}$ is ambiguous: it is a stochastic variable ranging between $T_{H}$ and $T_{L}$ which are the limiting temperatures that characterize the transition.

We thank J. M. Sancho and A. Labarta for fruitful discussions. This work has received financial support from CICyT (Project No. MAT98-0315) and CIRIT (Project
No. 2000SGR00025). F. J. P. acknowledges financial support from DGICyT.

[1] Shape Memory Materials, edited by K. Otsuka and C. M. Wayman (Cambridge University Press, Cambridge, 1998).

[2] Z. Nishiyama, Martensitic Transformations (Academic Press, New York, 1978), p. 240.

[3] For a recent review see A. Planes and Ll. Mañosa, Solid State Phys. 55, 159 (2001).

[4] G. Bertotti, Hysteresis in Magnetism (Academic Press, San Diego, 1998).

[5] E. Vives, J. Ortín, Ll. Mañosa, I. Ràfols, R. Pérez-Magrané, and A. Planes, Phys. Rev. Lett. 72, 1694 (1994); Ll. Carrillo, Ll. Mañosa, J. Ortín, A. Planes, and E. Vives, Phys. Rev. Lett. 81, 1889 (1998).

[6] Z. Yu and P. C. Clapp, J. Appl. Phys. 62, 2212 (1987); Ll. Mañosa, A. Planes, D. Rouby, M. Morin, P. Fleischmann, and J. L. Macqueron, Appl. Phys. Lett. 54, 2574 (1989).

[7] R. Vergne, J. C. Cotillard, and J. L. Porteseil, Rev. Phys. Appl. 16, 847 (1981).

[8] M. P. Lilly, A. H. Wootters, and R. B. Hallock, Phys. Rev. Lett. 70, 3347 (1995).

[9] For a recent review on crackling noise in complex systems see J.P. Sethna, K. A. Dahmen, and C. R. Myers, Nature (London) 410, 242 (2001).

[10] J. P. Sethna, K. Dahmen, S. Kartha, J. A. Krumhansl, B. W. Roberts, and J. D. Shore, Phys. Rev. Lett. 70, 3347 (1993).

[11] W. Cao, J. A. Krumhansl, and R. J. Gooding, Phys. Rev. B 41, 11319 (1990); M. Rao and S. Sengupta, Phys. Rev. Lett. 78, 2168 (1997).

[12] M. Aspelmeyer, U. Klemradt, L. T. Wood, S. C. Moss, and J. Peisl, Phys. Status Solidi (a) 174, R9 (1999); T. Kakeshita, T. Saburi, and K. Shimizu, Philos. Mag. B 80, 171 (2000).

[13] D. A. Vul and B. N. Harmon, Phys. Rev. B 48, 6880 (1993).

[14] The temperature was measured on the $\mathrm{Cu}$ block. The thermal lag between sample and block temperatures was calibrated for all the temperature rates investigated. A linear dependence of the lag on $\dot{T}$ has been found consistently with the predictions of an $R C$ model for heat conduction. See E. Calvet and H. Prat, Recent Progress in Microcalorimetry (Pergamon Press, New York, 1963).

[15] The recorded number of counts as a function of time has been double checked by using a continuous analog counter, connected in parallel with the frequency counter. Results are perfectly comparable which means that the dead times inherent to the gated frequency counter are irrelevant for the analysis.

[16] M. C. Torrent and M. San Miguel, Phys. Rev. A 38, 245 (1988); V. Pérez-Muñuzurri, F. Sagués, and J. M. Sancho, Phys. Rev. E 62, 94 (2000).

[17] J. L. Pelegrina and M. Ahlers, Acta Metall. Mater. 38, 293 (1990). 